\title{
CONTROLE DE Atta laevigata (HYMENOPTERA: FORMICIDAE) COM A ISCA LANDRIN-F, EM ÁREA ANTERIORMENTE COBERTA COM Eucalyptus
}

\author{
CONTROL OF Atta laevigata (HYMENOPTERA: FORMICIDAE), WITH \\ LANDRIN-F BAIT, IN AREAS PREVIOUSLY COVERED WITH Eucalyptus
}

\author{
José Cola Zanuncio $^{1}$ Teresinha Vinha Zanuncio $^{2}$ \\ José Milton Milagres Pereira ${ }^{3}$ Harley Nonato de Oliveira ${ }^{4}$
}

RESUMO

\begin{abstract}
Embora muitos produtos tenham sido testados como princípio ativo de iscas formicidas, o mercado predominantemente utilizava produtos à base de dodecacloro. Com a proibição deste último composto, surgiram as iscas à base de sulfluramida ou de clorpirifós, constituindo, atualmente, dois dos princípios ativos de iscas formicidas, disponíveis no mercado. Este trabalho objetivou testar a isca Landrin-F (clorpirifós 0,45\%) no controle da formiga cortadeira Atta laevigata ( $F$. Smith) (Hymenoptera: Formicidae), comparado à isca Mirex-S (sulfluramida 0,3\%). Foram utilizados tratamentos com a aplicação de 30 a 120 gramas da isca Landrin-F por olheiro; aplicação de $8 \mathrm{~g} / \mathrm{m}^{2}$ de formigueiro da isca Landrin-F; aplicação de $8 \mathrm{~g} / \mathrm{m}^{2}$ de formigueiro da isca Mirex-S e; testemunha, sem aplicação de isca formicida. Após 24 e 48 horas, avaliaram-se o transporte elou devolução das iscas, e aos 7, 15, 30,60,90,120,150, 180 e 210 dias, a atividade dos formigueiros, os quais foram sondados e escavados, aos 180 dias, e feita a avaliação final de atividade aos 210 dias. A utilização da metodologia de aplicação da isca Landrin-F por olheiro ativo aumentou consideravelmente a quantidade de isca aplicada, em relação à metodologia de aplicação por metro quadrado de formigueiro. Como a isca Landrin-F mostrou eficiência semelhante nas duas metodologias de aplicação, recomenda-se que a mesma seja aplicada na dosagem de $8 \mathrm{~g} / \mathrm{m}^{2}$ de formigueiro para o controle de A. laevigata.
\end{abstract}

Palavras-chave: controle químico, formigas cortadeiras, Eucalyptus.

\section{SUMMARY}

Although many insecticides have been tested as baits against leaf cutting ants, the market was dominated by those with dodecachlor. This compound was banned and the new baits for these pests have either sulfluramid or chlorpirifos. The objective of this work was to test the Landrin-F (chlorpirifos 0.45\%) bait against the leaf cutting ant Atta laevigata (F. Smith, 1858) (Hymenoptera: Formicidae) compared to Mirex-S (sulfluramid $0.3 \%)$ bait. Treatments used were: application of 30 to 120 grams of the Landrin-F bait for each ant hole; application of $8 \mathrm{~g} / \mathrm{m}^{2}$ of ant nest of Landrin-F bait; application of $8 \mathrm{~g} / \mathrm{m}^{2}$ of ant nest of Mirex-S bait and; control, no bait was applied. After 24 and 48 hours the transport and refusal of the baits were evaluated, and after $7,15,30,60,90,120,150,180$ and 210 days ant colonies activity was evaluated. Ant colonies were excavated after 180 days and its activity was evaluated after 210 days. The application of Landrin-F bait based in active ant holes increased the amount of bait in relation to bait quantity applied per square meter of ant colony. Since the efficiency of the Landrin-F bait was similar in both methodologies it is recomended that this bait should be applied at the dosage of $8 \mathrm{~g} / \mathrm{m}^{2}$ of ant colony to control A. laevigata.

Key words: chemical control, leaf-cutting ants, Eucalyptus.

\section{INTRODUÇÃO}

As formigas cortadeiras dos gêneros Atta e Acromyrmex (DELLA LUCIA et al., 1993) representam os maiores problemas entomológicos para várias culturas, incluindo os reflorestamentos com eucalipto, onde são responsáveis por danos consideráveis. Em razão disso, todas as empresas florestais possuem programas de controle desses insetos, com a utilização de diversos métodos, incluindo a termonebulização (COUTO et al., 1977), mas com desta-

\footnotetext{
${ }^{1}$ Engenheiro Florestal, PhD., Professor Titular do Departamento de Biologia Animal da Universidade Federal de Viçosa (UFV). 36571 000 - Viçosa, MG. E-mail: zanuncio@mail.ufv.br.

${ }^{2}$ Biólogo, Ms., Doutorando em Entomologia (UFV).

${ }^{3}$ Engenheiro Florestal, Ms., Pesquisador (UFV).

${ }^{4}$ Engenheiro Agrônomo, Ms., Doutorando em Entomologia (UFV). 
que para as iscas granuladas (ZANUNCIO et $\boldsymbol{a l}$., 1980). A. laevigata, conhecida como saúva cabeça de vidro ou de melado, pode ser considerada como a segunda espécie mais comum no Brasil, tendo ampla distribuição pelo interior do país. Seus ninhos se caracterizam por montes arredondados de terra solta, com a superfície quase sempre livre e os olheiros abrindo-se sobre esses montes (ANJOS et al., 1998).

Embora um grande número de produtos tenha sido testado, como princípio ativo de iscas formicidas, o mercado foi dominado por aquelas à base de dodecacloro. Com a proibição desse composto, devido aos problemas ambientais causados pelo mesmo (ZANUNCIO et al., 1993), surgiram as iscas formicidas à base de clorpirifós ou de sulfluramida. Em razão disso, muitos testes têm sido efetuados com essas iscas, nas mais diversas condições, visando a sua utilização para o controle de formigas cortadeiras. A eficiência de controle de iscas à base de sulfluramida é relatada para Acromyrmex subterraneus molestans (Santschi) (ZANUNCIO et al. 1996a), Atta laevigata (F. Smith) (ALVES et al. 1997) e Atta cephalotes (L.) (ZANUNCIO et al. 1996b). Outros estudos mostram a eficiência de iscas com sulfluramida ou clorpirifós para Atta sexdens sexdens (L.) (CRUZ et al. 1996) e para Acromyrmex laticeps nigrosetosus (Forel) (OLIVEIRA e ZANUNCIO, 1997).

Este trabalho objetivou testar a eficiência da isca Landrin-F (clorpirifós $0,45 \%$ ) no controle da formiga cortadeira A. laevigata, em duas metodologias de aplicação, comparada à isca Mirex-S (sulfluramida $0,3 \%$ ).

\section{MATERIAL E MÉTODOS}

Este ensaio foi realizado em área de reflorestamento na fazenda Buenos Aires I, da PLANTAR S.A., na região de Curvelo, Minas Gerais, em área coberta, anteriormente, por eucalipto, e que apresentava grande número de formigueiros, com predominância de $\boldsymbol{A}$. laevigata. Foram utilizados 60 formigueiros dessa espécie, com área média de $31,95 \mathrm{~m}^{2}$, com 15 deles para cada um dos seguintes tratamentos: (T1) em cada um dos formigueiros mediam-se áreas de $1,5 \mathrm{~m} \times 1,5 \mathrm{~m}$, onde cada olheiro isolado ou grupo de olheiros presentes foram divididos em três classes de tamanho, pequenos, médios e grandes, recebendo 30, 60 e 120 gramas da isca Landrin-F, respectivamente. Essa dosagem por olheiro segue metodologia padrão recomendada pela empresa produtora para a aplicação da isca LandrinF; (T2) aplicação de $8 \mathrm{~g} / \mathrm{m}^{2}$ de formigueiro, da isca Landrin-F; (T3) aplicação de $8 \mathrm{~g} / \mathrm{m}^{2}$ de formigueiro, da isca Mirex-S (sulfluramida) e (T4) testemunhasem aplicação de isca formicida.
Para que todos os tratamentos recebessem formigueiros de tamanhos semelhantes, os ninhos de A. laevigata foram distribuídos em três classes de tamanho (pequenos, médios e grandes). A seguir, os 20 formigueiros de cada classe foram sorteados, com cinco deles por tratamento. Dessa forma, o experimento foi instalado com 15 repetições, cada uma delas constituída por um formigueiro, sendo cinco de cada classe de tamanho.

Este ensaio foi instalado em 09 de julho de 1996, com avaliações de transporte (\% de iscas que foram carregadas em cada ponto) e/ou devolvidas após 24 e 48 horas, enquanto aos $7,15,30,60$, $90,120,150,180$ e 210 dias, avaliou-se a atividade dos formigueiros. Aos 180 dias, os formigueiros foram sondados e escavados e aos 210 dias, foi feita a avaliação final, para saber se existiam movimentação externa de formigas, transporte de terra solta e/ou corte de folhas.

Os dados de eficiência (\% de formigueiros que estavam paralisados aos 210 dias após a aplicação das iscas) dos tratamentos foram submetidos ao teste de Tukey em nível de 5\% de probabilidade.

\section{RESULTADOS E DISCUSSÃO}

A maior percentagem de isca transportada $(97,07 \%)$ ocorreu no tratamento T3, que recebeu a isca Mirex-S, a $8 \mathrm{~g} / \mathrm{m}^{2}$ de formigueiro, enquanto a isca Landrin-F, nessa mesma dosagem (tratamento T2) apresentou $87,73 \%$ de transporte. No tratamento T1 (isca Landrin-F de acordo com o tamanho do olheiro ativo), foram transportadas $67,50 \%$ da isca aplicada, o que pode ser devido ao fato da quantidade de isca nesse tratamento ter sido muito alta, em média 24,94 gramas $/ \mathrm{m}^{2}$ de formigueiro. De modo geral, as iscas Landrin-F e Mirex-S tiveram boa aceitação, com taxas de devolução de, apenas, 2,71; 2,07 e 2,00\% nos tratamentos T1, T2 e T3, respectivamente (tabela 1 ).

Aos sete dias, todos os formigueiros de $\boldsymbol{A}$. laevigata, que receberam a isca Landrin-F (tratamentos T1 e T2), estavam inativos, o que persistiu na avaliação aos 15 dias, enquanto os formigueiros tratados com a isca Mirex-S apresentavam, ainda, movimentação de formigas aos sete e 15 dias (tabela 2). Todos os formigueiros tratados com a isca Mirex-S estavam paralisados aos 60 dias, o que concorda com ALVES et al. (1997) para A. laevigata, pois, segundo esses autores, a aplicação dessa isca a $8 \mathrm{~g} / \mathrm{m}^{2}$ de formigueiro promove paralisação total de movimentação de formigas em até 60 dias após sua aplicação. Este período pode ser considerado longo, principalmente 
Tabela 1 - Área média e isca aplicada por metro quadrado de formigueiro, percentagem de transporte e devolução, número de formigueiros mortos e vivos e eficiência das iscas Landrin-F (clorpirifós 0,45\%) e Mirex-S (sulfluramida 0,3\%). Curvelo, Minas Gerais.

\begin{tabular}{|c|c|c|c|c|c|c|c|}
\hline \multirow[t]{2}{*}{$\begin{array}{l}\text { Trata- } \\
\text { mento }\end{array}$} & \multirow[t]{2}{*}{$\begin{array}{l}\text { Área } \\
\left(\mathrm{m}^{2}\right)\end{array}$} & \multirow[t]{2}{*}{$\begin{array}{r}\text { Isca/ } \\
\mathrm{m}^{2}\end{array}$} & \multirow[t]{2}{*}{$\begin{array}{c}\text { Trans- } \\
\text { porte }(\%)\end{array}$} & \multirow[t]{2}{*}{$\begin{array}{l}\text { Devolu- } \\
\text { ção }(\%)\end{array}$} & \multicolumn{2}{|c|}{$\begin{array}{l}\text { Número de Formi- } \\
\text { gueiros }\end{array}$} & \multirow[t]{2}{*}{$\begin{array}{c}\text { Efici- } \\
\text { ência (\%) }\end{array}$} \\
\hline & & & & & Mortos & Vivos & \\
\hline $\mathrm{T} 1$ & 27,64 & 24,93 & 67,50 & 2,71 & 17 & $2 *$ & $85,71^{\mathrm{a}}$ \\
\hline $\mathrm{T} 2$ & 36,27 & 8,00 & 87,73 & 2,07 & 17 & 3 & $80,00^{\mathrm{a}}$ \\
\hline $\mathrm{T} 3$ & 32,47 & 8,00 & 97,07 & 2,00 & 18 & 2 & $86,67^{\mathrm{a}}$ \\
\hline $\mathrm{T} 4$ & 31,40 & - & - & - & 0 & 20 & - \\
\hline
\end{tabular}

T1 - Landrin-F, aplicada de 30 a 120 gramas por olheiro ativo ou grupo de olheiros próximos. T2Landrin-F, a $8 \mathrm{~g} / \mathrm{m}^{2}$ de terra solta de formigueiro. T3- Mirex-S, a $8 \mathrm{~g} / \mathrm{m}^{2}$ de terra solta de formigueiro. T4- testemunha.

* Um formigueiro não foi localizado nesse tratamento.

Valores percentuais seguidos de mesma letra não diferem entre si pelo teste de Tukey, em nível de $5 \%$ de probabilidade.

se comparado à isca Landrin-F na mesma dosagem que mostrou paralisação de $100,00 \%$ dos formigueiros aos sete dias. Os resultados de paralisação mais rápida de formigueiros, tratados com a isca à base de clorpirifós, concordam com os apresentados por CRUZ et al. (1996), para $\boldsymbol{A}$. sexdens sexdens. No entanto, como o importante é a paralisação de corte, ALVES et al. (1997) mostraram que a atividade de corte e forrageamento de formigueiros de A. laevigata, tratados com a isca Mirex-S, praticamente cessa ao redor de seis dias, mesmo com movimentação de formigas na parte externa dos ninhos.

$\mathrm{Na}$ avaliação final, a eficiência da isca Landrin-F (tratamentos T1 e T2) foi de 85,71 e $80,00 \%$, respectivamente, semelhante àquela apresentada pela isca Mirex-S, que foi de $86,67 \%$, nessa última dosagem (tabela 2). Estes resultados demonstram que a isca Landrin-F pode ser aplicada de acordo com a dosagem por metro quadrado de formigueiro pois, desta forma, pode-se controlar A. laevigata com menor quantidade de isca. CRUZ et al. (1996), usando três iscas à base de clorpirifós, encontraram eficiência máxima de $44,4 \%$ no controle de $A$. sexdens sexdens, na região norte do Brasil. No entanto, LINK (1997) relata controle de 100,00\% para Atta sexdens piriventris (Santschi) (Hymenoptera: Formicidae), com a isca Landrin-F na dosagem de $10 \mathrm{~g} / \mathrm{m}^{2}$ e de $75,00 \%$ para a isca Mirex à base de dodecacloro.
Resultados conflitantes, da eficiência de iscas à base de clorpirifós, demonstram que elas necessitam ter qualidade muito alta, o que tem sido conseguido pela isca Landrin-F, que dá uma melhor proteção a esse princípio ativo. Por isso, essa isca tem apresentado maior eficiência que outras com esse mesmo ingrediente ativo.

A aplicação da isca Landrin-F, por olheiro ativo ou grupo de olheiros próximos, aumentou consideravelmente a quantidade de isca aplicada, em relação à metodologia tradicional de aplicação. Por isso, novos estudos devem ser efetuados visando à redução do total de isca nesse método, pois o mesmo pode apresentar maior eficiência que o tradicional, em razão da melhor distribuição do produto sobre o formigueiro. Por outro lado, as sobras de isca, na aplicação por olheiro isolado ou grupo de olheiros próximos, demonstram que essa dosagem pode ser reduzida em, pelo menos, 30,00\%, para A. laevigata. Com isso, a isca Landrin-F pode ser aplicada na dosagem de 30 a 80 gramas por olheiro isolado ou grupo de olheiros próximos. A melhoria desse método de aplicação de iscas é necessária, pois o mesmo tem utilidade especial em situações que podem dificultar o dimensionamento do formigueiro (área de terra solta), como no cultivo mínimo ou em locais

Tabela 2 - Percentagem de paralisação da atividade externa de formigueiros que receberam as iscas Landrin-F (clorpirifós 0,45\%) e Mirex-S (sulfluramida $0,3 \%$ ). Curvelo, Minas Gerais.

\begin{tabular}{|c|c|c|c|c|c|}
\hline \multirow[t]{2}{*}{ Tratamento } & \multicolumn{5}{|c|}{ Paralisação (\%) } \\
\hline & 7 dias & 15 dias & 30 dias & 60 dias & 180 dias \\
\hline $\mathrm{T} 1$ & 100,00 & 100,00 & 100,00 & 92,80 & $85,71^{*}$ \\
\hline $\mathrm{T} 2$ & 100,00 & 100,00 & 93,33 & 93,33 & 80,00 \\
\hline T3 & 80,00 & 86,67 & 66,67 & 100,00 & 86,67 \\
\hline $\mathrm{T} 4$ & - & - & - & - & - \\
\hline
\end{tabular}

T1- Landrin-F, aplicada de 30 a 120 gramas por olheiro ativo ou grupo de olheiros próximos. T2- Landrin-F, a $8 \mathrm{~g} / \mathrm{m}^{2}$ de terra solta de formigueiro. T3- Mirex-S, a $8 \mathrm{~g} / \mathrm{m}^{2}$ de terra solta de formigueiro. T4- testemunha.

* Um formigueiro não foi localizado nesse tratamento. 
onde o solo é revolvido, como em áreas de cana-deaçúcar.

Recomenda-se que novas pesquisas sejam feitas visando à redução da quantidade de isca na metodologia de dose por olheiro ou grupo de olheiros próximos. A melhoria dessa metodologia é importante pois a quantidade de isca irá variar com o número, tamanho e atividade dos olheiros, os quais dependem da atividade interna do sauveiro. Finalmente, o ajuste dessa metodologia pode torná-la mais adequada e com melhor eficiência para o controle de formigas cortadeiras.

\section{CONCLUSÃO}

A isca Landrin-F pode ser aplicada na dosagem de oito gramas por metro quadrado de formigueiro para o controle de A. laevigata.

\section{AGRADECIMENTOS}

Ao CNPq, CAPES e FAPEMIG, pelas bolsas e auxílios e ao Sr. Geraldo M. Rodrigues, da Universidade Federal de Viçosa, pelo auxílio durante a realização deste trabalho.

\section{REFERÊNCIAS BIBLIOGRÁFICAS}

ALVES, J.B., ZANUNCIO, J.C., GALO, M.V., et al. Paralisação de forrageamento e controle de Atta laevigata (F. Smith) (Hymenoptera: Formicidae) com Mirex-S (sulfluramida) em duas metodologias de medição de formigueiros. Revista Árvore, v. 21, n 1, p. 141-146, 1997.

ANJOS, N., DELlA LUCIA, T.M.C., MAYHÉ-NUNES, A.J. Guia prático sobre formigas cortadeiras em reflorestamentos. Ponte Nova, MG.: Graffcor, 1998. 97 p.

COUTO, L., ZANUNCIO, J.C., ALVES, J.E., et al. Avaliação da eficiência de controle de Atta sexdens rubropilosa através do sistema de termonebulização, na Aracruz, Espírito Santo. Revista Árvore, v. 1, n. 1, p. 9-16, 1977.
CRUZ, A.P., ZANUNCIO, J.C., ZANETTI, R., et al. Eficiência de iscas granuladas à base de sulfluramida e de clorpirifós no controle de Atta sexdens sexdens (Hymenoptera: Formicidae), no trópico úmido. Acta Amazonica, v. 26, n. 3, p. 145-150, 1996.

DELLA LUCIA, T.M.C., FOWLER, H.G., MOREIRA, D.D.O Espécies de formigas cortadeiras no Brasil. In: T.M.C. DELlA LUCIA (ed.). As Formigas Cortadeiras. Viçosa, MG.: Folha de Viçosa, 1993. p. 26-31.

LINK, D. Controle da formiga saúva limão sulina, Atta sexdens piriventris, com novas iscas granuladas. In: XII ENCONTRO DE MIRMECOLOGIA. Ilhéus, BA. Anais... Ilhéus: Universidade Estadual de Santa Cruz, 1997. 190 p. p. 160.

OLIVEIRA, H.N., ZANUNCIO, J.C. Controle de Acromyrmex laticeps nigrosetosus Forel, 1908 (Hymenoptera: Formicidae), no trópico úmido, com iscas granuladas à base de sulfluramida e de clorpirifós. In: VI INTERNATIONAL PEST ANT SYMPOSIUM \& XIII ENCONTRO DE MIRMECOLOGIA, 1997. Ilhéus, Ba. Anais... Ilhéus: Universidade Estadual de Santa Cruz 1997. 190 p. p. 169.

ZANUNCIO, J.C., VILELA, E.F., NOGUEIRA. S.B. Emprego de iscas granuladas e pós secos no controle de Atta laevigata, no município de Curvelo, MG. Revista Árvore, v. 4, n. 2, p. 221-226, 1980.

ZANUNCIO, J.C., ZANUNCIO, T.V., SANTOS, G.P. A contribuição da pesquisa, em entomologia florestal, para a redução dos impactos ambientais dos reflorestamentos. In: SIMPÓSIO BRASILEIRO DE PESQUISA FLORESTAL, 1993. Belo Horizonte, MG. Anais... Viçosa: Folha de Viçosa,, 1993. v. 1. p. 136-142.

ZANUNCIO, J.C., LARANJEIRO, A.J., De SOUZA O. Controle de Acromyrmex subterraneus molestans Santschi (Hymenoptera: Formicidae) com sulfluramida. An Soc Entomol Brasil, v. 25, n. 3, p. 383-388, 1996 a.

ZANUNCIO, J.C., CRUZ, A.P., SANTOS, D.F., et al. Eficiência da isca Mirex-S (sulfluramida 0,3\%) no controle de Atta cephalotes (Hymenoptera: Formicidae) em três dosagens. Acta Amazonica, v. 26, n. 1/2, p. 115-120, 1996 b. 